\title{
CONTENIDO POLÍNICO DE LA ATMÓSFERA DE MÁLAGA: AÑO 1994
}

\author{
Marta RECIO, M. Mar TRIGO, F. Javier TORO y Baltasar CABEZUDO
}

RESUMEN. Contenido polínico de la atmósfera de Málaga: año 1994. Con el presente trabajo se pretende dar a conocer los resultados obtenidos tras el muestreo aerobiológico realizado en Málaga a lo largo del año 1994. Por medio de un muestreador tipo Hirst, de la casa Burkard $®$, se ha analizado la evolución estacional del contenido polínico de la atmósfera, comparando estos resultados con los datos obtenidos durante los dos años anteriores. Asimismo se señalan cuáles fueron los periodos de máxima concentración de los taxones que más incidencia tuvieron en la atmósfera de la ciudad.

Palabras clave. Aerobiología, polen, Málaga, sur de España.

ABSTRACT. Pollen analysis content in the atmosphere of Málaga: 1994. During 1994 a pollen content analysis of the atmosphere has been carried out in Málaga (southern Spain) with the aim to release the obtained results. The sampling was made with the aid of a Burkard spore-trap (Hirst method) located on the roof of the Faculty of Medicine of Málaga University, about $15 \mathrm{~m}$ obove ground level. The pollen data, expresed in grains per cubic metre, were obtained counting four lengths of the slide with a 40X objective $(0,45 \mathrm{~mm}$ field). During this year, we have observed that about $90 \%$ of total pollen was collected from the last week of January to June, with two maximum peaks in April and May, months that gets together about $50 \%$ out of total annual. On the contrary, the lowest monthly concentration was detected from July to December. Along this period, the most important taxa in the atmosphere of Málaga were Cupressaceae and Olea europaea followed by Quercus, Poaceae, Chenopodiaceae-Amaranthaceae, Plantago, Urticaceae and Casuarina, in importance order. So, the different peaks that occur along the year are fundamentally due, to Cupressaceae in January-February; Cupressaceae, Pinus and Platanus in February; Quercus, Plantago and Chenopodiaceae-Amaranthaceae in April; Olea and Poaceae in May and Casuarina in October.

Key words. Aerobiology, pollen, Málaga, southern Spain.

\section{INTRODUCCIÓN}

En un reciente estudio llevado a cabo en todo el territorio español, se ha puesto de manifiesto la elevada incidencia del polen en los pacientes afectados de sintomatología alérgica, de tal forma que, en la zona sur (Andalucía y Extremadura) alrededor del 50\% de estos pacientes, presentan sensibilidad a algún tipo de polen (S.E.A.I.C. y Abelló, S.A., eds., 1995). Según dicho estudio, la mayoría (más del 60\%) de estos pacientes afectados de 
polinosis vivían en el medio urbano, de ahí la importancia de realizar un muestreo continuado de la atmósfera de estas zonas urbanas con objeto de poder establecer un calendario polínico así como modelos de comportamiento estacional más o menos estables. Concretamente en Málaga, según García González (1995), son los pólenes de olivo y de gramíneas, seguidos de los de Chenopodium y Parietaria, los que más incidencia tienen en la población.

Continuando con los datos ya publicados anteriormente sobre los años 1992 y 1993 (Cabezudo et al., 1994), en el presente trabajo se incluyen los resultados más significativos, obtenidos durante el año 1994, del contenido polínico de la atmósfera de Málaga, con objeto de dar a conocer el comportamiento que los distintos taxones han presentado a lo largo del año.

\section{MATERIAL Y MÉTODOS}

El muestreo se ha realizado utilizando un captador volumétrico tipo Hirst de la casa Burkard ${ }^{\circledR}$ colocado en el tejado de la Facultad de Medicina de La Universidad de Málaga, Campus de Teatinos, a unos $15 \mathrm{~m}$ aproximadamente del nivel del suelo. Este edificio se encuentra situado a $1 \mathrm{~km}$ al oeste de la ciudad, en un espacio abierto y sin edificios colindantes que pudieran impedir la libre circulación del aire.

El recuento se ha llevado a cabo según la metodología propuesta por Domínguez et al. (1991) y adoptada por la Red Española de Aerobiología (REA), habiéndose realizado 4 barridos por preparación, utilizándose como medio de montaje glicerogelatina coloreada con fucsina básica y como sustancia adhesiva petrolato blanco. Todos los datos vienen expresados en granos de polen por metro cúbico de aire $\left(\right.$ granos $\left./ \mathrm{m}^{3}\right)$. Para la elaboración de tablas y gráficas se ha trabajado a partir de los valores medios diarios. Los datos meteorológicos han sido facilitados por el Centro Meteorológico Zonal de Málaga y corresponden a la estación Málaga-Aeropuerto, situada a unos $5 \mathrm{~km}$ al suroeste del punto de muestreo.

\section{RESULTADOS}

Durante el año 1994 se observa que las mayores concentraciones se obtuvieron desde finales de Enero a finales de Junio, periodo en el que se concentró casi el $90 \%$ del polen total anual, mientras que las concentraciones más bajas se registraron de Julio a Diciembre, con tan sólo un pequeño pico destacable en octubre, debido fundamentalmente al aporte de polen de Casuarina (tabs. 1, 2 y 3; fig. 1).

\begin{tabular}{lcc}
\hline Meses & Total & $\%$ \\
\hline Enero & 1698 & 6,01 \\
Febrero & 2351 & 8,32 \\
Marzo & 3019 & 10,68 \\
Abril & 6731 & 23,82 \\
Mayo & 9010 & 31,89 \\
Junio & 2562 & 9,07 \\
Julio & 507 & 1,79 \\
Agosto & 220 & 0,78 \\
Septiembre & 345 & 1,22 \\
Octubre & 866 & 3,06 \\
Noviembre & 545 & 1,93 \\
Diciembre & 401 & 1,42 \\
\hline Total anual & 28255 & 100,00 \\
\hline
\end{tabular}

Tabla 1. Valores mensuales, absolutos (granos $/ \mathrm{m}^{3}$ ) y relativos (\%), obtenidos por las concentraciones polínicas a lo largo del año 1994. Monthly values (grains $/ \mathrm{m}^{3}$ ) and percentages (\%) obtained by the total pollen counts along 1994. 


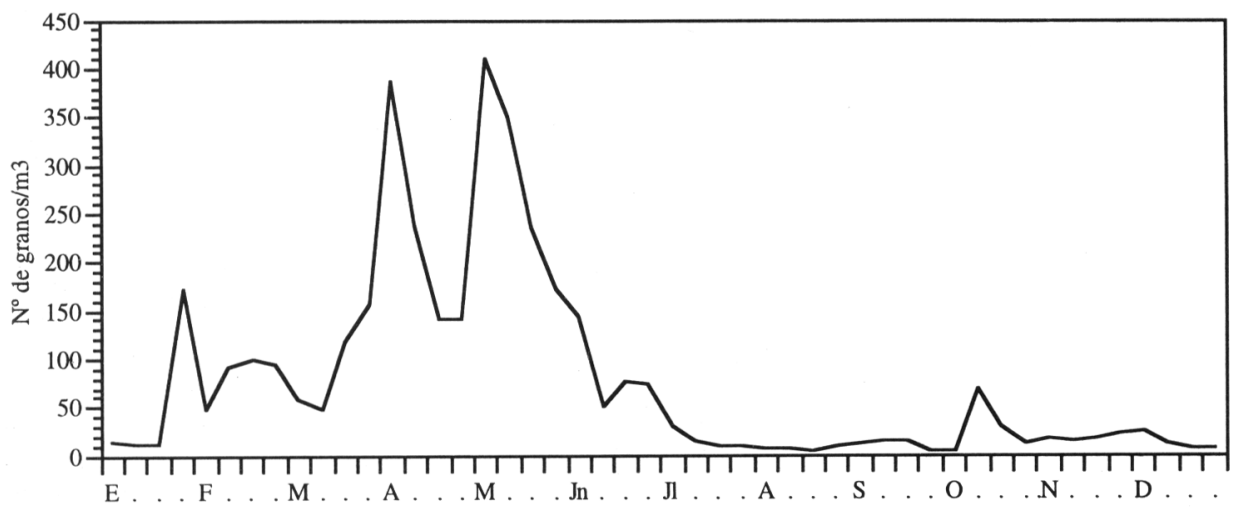

Figura 1. Evolución de la concentración media semanal del polen total registrada en la atmósfera de Málaga durante el año 1994. Total pollen concentration trend registred in the atmosphere of Málaga during 1994 (Week average).

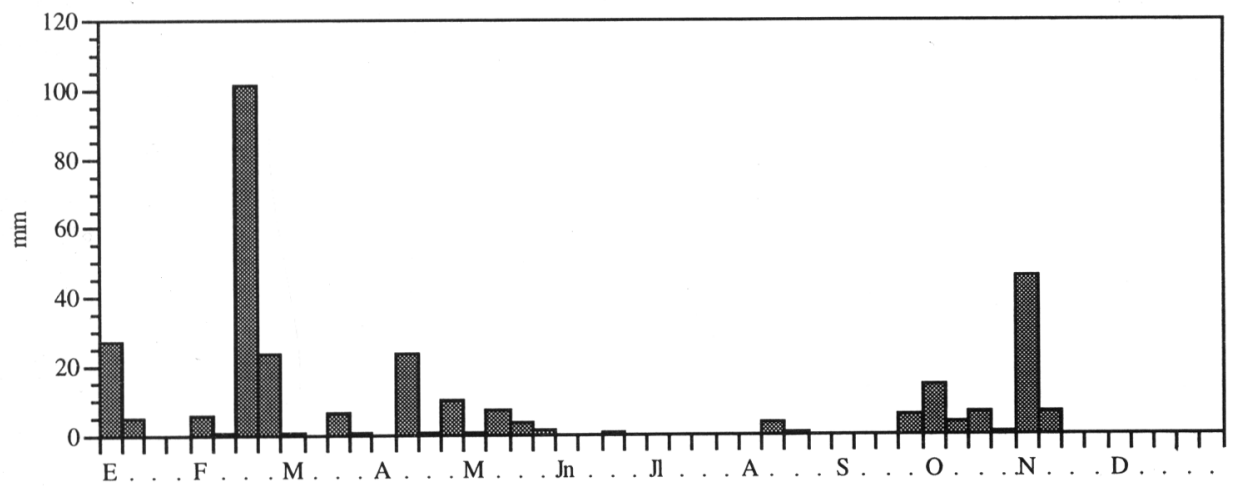

Figura 2. Precipitaciones totales semanales registradas en Málaga durante el año 1994. Week total rainfalls occur in Málaga during 1994.

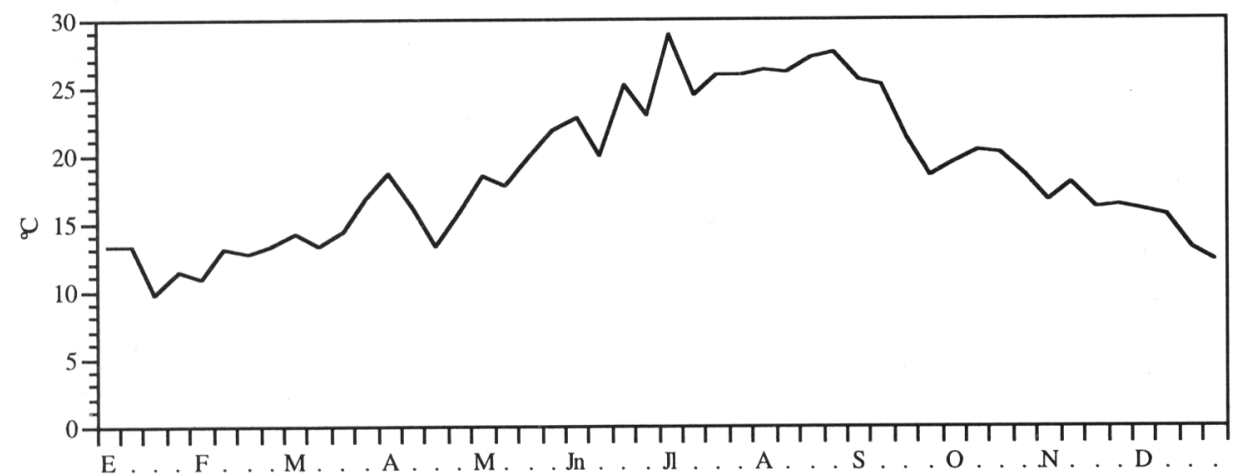

Figura 3. Evolución de la temperatura media semanal registrada en Málaga durante el año 1994. Week mean temperature trend registred in Málaga during 1994. 


\begin{tabular}{|c|c|c|c|c|c|c|c|c|c|c|c|c|c|}
\hline Taxón & Ene & $\mathrm{Feb}$ & Mar & Abr & May & Jun & Jul & Ago & Sep & Oct & Nov & Dic & Total anual \\
\hline Alnus & 11 & 4 & 1 & 0 & 0 & 0 & 0 & 0 & 0 & 0 & 0 & 1 & 17 \\
\hline Apiaceae & 0 & 0 & 1 & 10 & 26 & 57 & 11 & 3 & 5 & 2 & 14 & 0 & 129 \\
\hline Artemisia & 14 & 0 & 2 & 0 & 1 & 1 & 0 & 4 & 10 & 4 & 14 & 54 & 104 \\
\hline Cannabis & 0 & 0 & 0 & 0 & 10 & 7 & 9 & 4 & 0 & 0 & 0 & 0 & 30 \\
\hline Castanea & 1 & 1 & 1 & 4 & 3 & 61 & 18 & 2 & 2 & 0 & 0 & 0 & 93 \\
\hline Casuarina & 1 & 0 & 0 & 0 & 0 & 0 & 0 & 0 & 0 & 670 & 22 & 23 & 716 \\
\hline Compositae * & 2 & 8 & 12 & 50 & 55 & 58 & 18 & 12 & 22 & 18 & 7 & 4 & 266 \\
\hline Cupressaceae & 1378 & 1446 & 674 & 39 & 43 & 15 & 1 & 2 & 4 & 27 & 242 & 102 & 3973 \\
\hline Cyperaceae & 0 & 0 & 2 & 17 & 87 & 43 & 3 & 0 & 1 & 0 & 0 & 0 & 153 \\
\hline Chenop.-Amarant. & 3 & 5 & 79 & 753 & 476 & 119 & 49 & 68 & 106 & 22 & 7 & 6 & 1693 \\
\hline Echium & 0 & 0 & 9 & 28 & 26 & 2 & 0 & 0 & 0 & 0 & 0 & 0 & 65 \\
\hline Ericaceae & 0 & 7 & 2 & 6 & 19 & 1 & 0 & 0 & 1 & 0 & 1 & 1 & 38 \\
\hline Fraxinus & 13 & 11 & 4 & 1 & 0 & 0 & 0 & 0 & 0 & 0 & 9 & 3 & 41 \\
\hline Gramineae & 14 & 17 & 59 & 159 & 1254 & 462 & 47 & 24 & 34 & 15 & 4 & 9 & 2098 \\
\hline Ligustrum & 1 & 0 & 0 & 0 & 6 & 10 & 3 & 0 & 1 & 0 & 0 & 0 & 21 \\
\hline Mercurialis & 20 & 41 & 57 & 32 & 13 & 4 & 0 & 0 & 1 & 0 & 2 & 32 & 202 \\
\hline Morus & 1 & 2 & 21 & 8 & 1 & 0 & 0 & 0 & 0 & 0 & 0 & 0 & 33 \\
\hline Myrtaceae & 3 & 4 & 3 & 10 & 36 & 339 & 163 & 21 & 20 & 5 & 6 & 3 & 613 \\
\hline Olea & 1 & 1 & 61 & 1260 & 4737 & 538 & 16 & 9 & 10 & 2 & 1 & 1 & 6637 \\
\hline Palmae & 8 & 3 & 4 & 5 & 2 & 5 & 2 & 3 & 6 & 41 & 67 & 21 & 167 \\
\hline Parkinsonia & 0 & 0 & 0 & 0 & 0 & 44 & 18 & 11 & 2 & 0 & 0 & 0 & 75 \\
\hline Pinaceae & 1 & 378 & 100 & 74 & 27 & 29 & 3 & 4 & 2 & 0 & 0 & 1 & 619 \\
\hline Pistacia & 0 & 0 & 69 & 39 & 1 & 0 & 0 & 0 & 0 & 0 & 0 & 0 & 109 \\
\hline Plantago & 1 & 3 & 185 & 818 & 514 & 145 & 6 & 3 & 3 & 1 & 3 & 1 & 1683 \\
\hline Platanus & 0 & 3 & 141 & 11 & 3 & 3 & 0 & 0 & 0 & 0 & 0 & 0 & 161 \\
\hline Populus & 1 & 35 & 60 & 2 & 1 & 1 & 0 & 0 & 1 & 0 & 0 & 0 & 101 \\
\hline Quercus & 4 & 4 & 601 & 2092 & 449 & 35 & 9 & 0 & 1 & 0 & 6 & 3 & 3204 \\
\hline Ricinus & 6 & 5 & 10 & 9 & 12 & 4 & 2 & 1 & 1 & 3 & 14 & 13 & 80 \\
\hline Rumex & 5 & 12 & 50 & 156 & 206 & 39 & 1 & 1 & 1 & 0 & 0 & 1 & 472 \\
\hline Salix & 5 & 3 & 3 & 1 & 0 & 0 & 0 & 0 & 0 & 0 & 0 & 0 & 12 \\
\hline Typha & 0 & 0 & 0 & 0 & 9 & 58 & 2 & 0 & 0 & 0 & 0 & 0 & 69 \\
\hline Ulmus & 1 & 10 & 2 & 1 & 0 & 0 & 0 & 0 & 0 & 0 & 0 & 0 & 14 \\
\hline Urticaceae & 117 & 228 & 398 & 265 & 234 & 88 & 24 & 6 & 10 & 15 & 76 & 70 & 1531 \\
\hline Otros & 86 & 128 & 395 & 875 & 762 & 394 & 102 & 42 & 101 & 41 & 50 & 52 & 3028 \\
\hline
\end{tabular}

Tabla 2. Sumas mensuales y anuales de las concentraciones medias diarias de polen $\left(\right.$ granos $\left./ \mathrm{m}^{3}\right)$ obtenidas por los distintos taxones estudiados durante el año 1994. Monthly and annual sum of the daily average concentrations (grains $/ \mathrm{m}^{3}$ ) rise by the different taxa during 1994. * Excluido Artemisia. Excluding Artemisia.

Durante el período que abarca de Enero a Junio, se observan 5 picos importantes que reflejan distintas situaciones del contenido polínico de la atmósfera (fig. 1). Así, a finales de Enero se produjo un primer pico debido en algo más del $81 \%$ al polen de Cupresáceas, taxón que alcanzó su valor máximo diario el 29 de este mes (tab. 4). Posteriormente, ya en Febrero, tras un importante descenso de las concentraciones polínicas, se produjo un segundo pico debido, en gran parte, al polen de
Cupresáceas pero también al aumento del polen de Pinus y Urticáceas

Marzo supuso un período de transición en el que se observó un importante descenso del polen de Cupresáceas a la vez que aumentaban los de otros taxones como Quercus, Plantago y Chenopodiáceas-Amarantáceas, que alcanzarían sus picos máximos a lo largo del mes siguiente, siendo en este mes de Marzo cuando se obtuvieron las máximas concentraciones de polen de Urticáceas y Platanus. 


\begin{tabular}{|c|c|c|c|c|c|c|c|c|c|c|c|c|c|}
\hline Taxón & Ene & $\mathrm{Feb}$ & Mar & $\mathrm{Abr}$ & May & Jun & Jul & Ago & Sep & Oct & Nov & Dic & Total anual \\
\hline Alnus & 0,65 & 0,17 & 0,03 & 0,00 & 0,00 & 0,00 & 0,00 & 0,00 & 0,00 & 0,00 & 0,00 & 0,25 & 0,06 \\
\hline Apiaceae & 0,00 & 0,00 & 0,03 & 0,15 & 0,29 & 2,22 & 2,17 & 1,36 & 1,45 & 0,23 & 2,57 & 0,00 & 0,46 \\
\hline Artemisia & 0,82 & 0,00 & 0,07 & 0,00 & 0,01 & 0,04 & 0,00 & 1,82 & 2,89 & 0,46 & 2,57 & 13,47 & 0,37 \\
\hline Cannabis & 0,00 & 0,00 & 0,00 & 0,00 & 0,08 & 0,27 & 1,78 & 1,82 & 0,00 & 0,00 & 0,00 & 0,00 & 0,11 \\
\hline Castanea & 0,06 & 0,04 & 0,03 & 0,10 & 0,03 & 2,38 & 3,55 & 0,91 & 0,58 & 0,00 & 0,00 & 0,00 & 0,33 \\
\hline Casuarina & 0,06 & 0,00 & 0,00 & 0,00 & 0,00 & 0,00 & 0,00 & 0,00 & 0,00 & 77,37 & 4,04 & 5,74 & 2,53 \\
\hline Compositae * & 0,12 & 0,34 & 0,40 & 0,74 & 0,61 & 2,26 & 3,55 & 5,45 & 6,38 & 2,08 & 1,28 & 0,99 & 0,94 \\
\hline Cupressaceae & 81,15 & 61,51 & 22,33 & 0,58 & 0,48 & 0,59 & 0,19 & 0,91 & 1,16 & 3,12 & 44,40 & 25,44 & 14,06 \\
\hline Cyperaceae & 0,00 & 0,00 & 0,07 & 0,25 & 0,97 & 1,68 & 0,59 & 0,00 & 0,29 & 0,00 & 0,00 & 0,00 & 0,54 \\
\hline Chenop.-Amarant. & 0,18 & 0,21 & 2,62 & 11,19 & 5,28 & 4,64 & 9,66 & 30,91 & 30,72 & 2,54 & 1,28 & 1,50 & 5,99 \\
\hline Echium & 0,00 & 0,00 & 0,30 & 0,42 & 0,29 & 0,08 & 0,00 & 0,00 & 0,00 & 0,00 & 0,00 & 0,00 & 0,23 \\
\hline Ericaceae & 0,00 & 0,30 & 0,07 & 0,09 & 0,21 & 0,04 & 0,00 & 0,00 & 0,29 & 0,00 & 0,18 & 0,24 & 0,13 \\
\hline Fraxinus & 0,77 & 0,47 & 0,13 & 0,01 & 0,00 & 0,00 & 0,00 & 0,00 & 0,00 & 0,00 & 1,65 & 0,75 & 0,15 \\
\hline Gramineae & 0,82 & 0,72 & 2,00 & 2,36 & 13,90 & 18,03 & 9,27 & 10,91 & 9,86 & 1,73 & 0,73 & 2,24 & 7,43 \\
\hline Ligustrum & 0,06 & 0,00 & 0,00 & 0,00 & 0,07 & 0,39 & 0,59 & 0,00 & 0,29 & 0,00 & 0,00 & 0,00 & 0,07 \\
\hline Mercurialis & 1,18 & 1,74 & 1,90 & 0,48 & 0,14 & 0,16 & 0,00 & 0,00 & 0,29 & 0,00 & 0,37 & 7,98 & 0,71 \\
\hline Morus & 0,06 & 0,09 & 0,70 & 0,12 & 0,01 & 0,00 & 0,00 & 0,00 & 0,00 & 0,00 & 0,00 & 0,00 & 0,12 \\
\hline Myrtaceae & 0,18 & 0,17 & 0,10 & 0,15 & 0,39 & 13,23 & 32,15 & 9,55 & 5,80 & 0,58 & 1,10 & 0,75 & 2,17 \\
\hline Olea & 0,06 & 0,04 & 2,02 & 18,72 & 52,57 & 20,99 & 3,16 & 4,09 & 2,90 & 0,23 & 0,18 & 0,25 & 23,49 \\
\hline Palmae & 0,47 & 0,13 & 0,13 & 0,07 & 0,02 & 0,20 & 0,39 & 1,36 & 1,74 & 4,73 & 12,29 & 5,24 & 0,59 \\
\hline Parkinsonia & 0,00 & 0,00 & 0,00 & 0,00 & 0,00 & 1,71 & 3,55 & 5,00 & 0,58 & 0,00 & 0,00 & 0,00 & 0,26 \\
\hline Pinaceae & 0,06 & 16,08 & 3,31 & 1,10 & 0,30 & 1,13 & 0,59 & 1,82 & 0,58 & 0,00 & 0,00 & 0,25 & 2,19 \\
\hline Pistacia & 0,00 & 0,00 & 2,29 & 0,58 & 0,01 & 0,00 & 0,00 & 0,00 & 0,00 & 0,00 & 0,00 & 0,00 & 0,39 \\
\hline Plantago & 0,06 & 0,13 & 6,13 & 12,15 & 5,70 & 5,66 & 1,18 & 1,36 & 0,87 & 0,12 & 0,55 & 0,25 & 5,96 \\
\hline Platanus & 0,00 & 0,13 & 4,67 & 0,16 & 0,03 & 0,12 & 0,00 & 0,00 & 0,00 & 0,00 & 0,00 & 0,00 & 0,57 \\
\hline Populus & 0,06 & 1,49 & 1,99 & 0,03 & 0,01 & 0,04 & 0,00 & 0,00 & 0,29 & 0,00 & 0,00 & 0,00 & 0,36 \\
\hline Quercus & 0,24 & 0,17 & 19,91 & 31,01 & 4,98 & 1,37 & 1,78 & 0,00 & 0,29 & 0,00 & 0,00 & 0,75 & 11,34 \\
\hline Ricinus & 0,35 & 0,21 & 0,33 & 0,13 & 0,13 & 0,16 & 0,39 & 0,45 & 0,29 & 0,35 & 2,57 & 3,24 & 0,28 \\
\hline Rumex & 0,29 & 0,51 & 1,66 & 2,32 & 2,29 & 1,52 & 0,20 & 0,45 & 0,29 & 0,00 & 0,00 & 0,25 & 1,67 \\
\hline Salix & 0,29 & 0,13 & 0,10 & 0,01 & 0,00 & 0,00 & 0,00 & 0,00 & 0,00 & 0,00 & 0,00 & 0,00 & 0,04 \\
\hline Typha & 0,00 & 0,00 & 0,00 & 0,00 & 0,10 & 2,26 & 0,39 & 0,00 & 0,00 & 0,00 & 0,00 & 0,00 & 0,24 \\
\hline Ulmus & 0,06 & 0,42 & 0,07 & 0,01 & 0,00 & 0,00 & 0,00 & 0,00 & 0,00 & 0,00 & 0,00 & 0,00 & 0,05 \\
\hline Urticacea & 6,89 & 9,70 & 13,18 & 3,98 & 2,60 & 3,43 & 4,73 & 2,73 & 2,89 & 1,73 & 13,94 & 17,46 & 5,43 \\
\hline Suma & 94,94 & 94,90 & 86,57 & 86,91 & 91,50 & 84,60 & 79,86 & 80,90 & 70,72 & 95,27 & 89,70 & 87,04 & 89,26 \\
\hline
\end{tabular}

Tabla 3. Porcentajes mensuales y anuales de los taxones estudiados con respecto al polen total obtenido durante el año 1994. Monthly and annual percentage of the studied taxa in relation with the total pollen obtained during 1994. * Excluido Artemisia. Excluding Artemisia.

En los meses de Abril y Mayo, se concentró más del $50 \%$ del polen total anual, produciéndose a lo largo de este período los dos picos semanales más importantes del año; el primero de ellos obtenido durante la primera semana de Abril, debido en un elevado porcentaje al aporte de polen de Quercus, Plantago y Chenopodiáceas-Amarantáceas; y el segundo, ligeramente mayor, durante la primera semana de Mayo, propiciado en este caso por el polen de olivo y de gramíneas fundamentalmente, que alcanzaron el día 8 los valores máximos diarios (tab. 4).

Hacia finales de Mayo, las concentraciones de polen descendieron sensiblemente, observándose un ligero incremento hacia la segunda quincena de Junio debido a la aparición de taxones típicamente estivales como Mirtáceas, Apiáceas, Castanea, Parkinsonia, Cyperáceas y Typha, aunque, por otra parte, aún se detectaron concentraciones importantes de polen de olivo y de gramíneas, especialmente 


\begin{tabular}{|l|cc|cc|}
\hline Taxón & Día pico & $\begin{array}{c}\text { Concentración } \\
\text { media diaria }\end{array}$ & Semana pico & $\begin{array}{c}\text { Concentración } \\
\text { media semanal }\end{array}$ \\
\hline Cupressaceae & 29 Enero & 602 & $4^{\text {a }}$ Enero & 163 \\
Pinaceae & 14 Febrero & 59 & $2^{\text {a }}$ Febrero & 22 \\
Urticaceae & 21 Marzo & 47 & $3^{\text {a }}$ Marzo & 19 \\
Quercus & 7 Abril & 261 & $1^{\text {a }}$ Abril & 165 \\
Plantago & 7 Abril & 95 & $1^{\text {a }}$ Abril & 55 \\
Chenop.-Amar. & 27 Abril & 73 & $4^{\text {a }}$ Abril & 30 \\
Olea europaea & 8 Mayo & 1060 & $1^{\text {a } \text { Mayo }}$ & 235 \\
Gramineae & 8 Mayo & 149 & $2^{\text {a } \text { Mayo }}$ & 69 \\
Rumex & 8 Mayo & 21 & $1^{\text {a Abril }}$ & 12 \\
Myrtaceae & 25 Junio & 112 & $4^{\text {a Junio }}$ & 23 \\
Compuestas* & 26 Junio & 14 & $4^{\text {a Junio }}$ & 3 \\
Casuarina & 10 Octubre & 221 & $2^{\text {a } \text { Octubre }}$ & 66 \\
\hline
\end{tabular}

Tabla 4. Valores máximos obtenidos por los 12 taxones más relevantes en la atmósfera de Málaga durante el año 1994. Maximum values obtained by the 12 more important taxa in the atmosphere of Málaga during 1994 (day and week peak). * Excluido Artemisia. Excluding Artemisia.

durante la primera semana de Junio.

Julio, Agosto y Septiembre, fueron meses en los que se obtuvieron valores mensuales muy bajos (tab. 1), y en los que sólo cabe destacar la presencia de polen de eucalipto, gramíneas, Chenopodiáceas-Amarantáceas y algunas compuestas, aunque en cantidades poco importantes.

En el mes de Octubre se produjo un último pico, debido, en algo más del $77 \%$, al polen de Casuarina, mientras que Noviembre y Diciembre fueron, al igual que Julio, Agosto y Septiembre, meses de baja concentración polínica, si bien cabe destacar un ligero incremento de polen de Urticáceas y Cupresáceas, y cierta importacia relativa del de Artemisia, Palmáceas, Mercurialis y Ricinus.

En cuanto a los taxones que mayor incidencia tuvieron en la atmósfera de Málaga durante el año 1994, se observa (tab. 3) que, en orden de abundancia anual, fueron olivo $(23,49 \%)$ y Cupresáceas $(14,06 \%)$, seguidos de Quercus $(11,34 \%)$, gramíneas $(7,43 \%)$, Chenopodiáceas-Amarantáceas $(5,99 \%)$, Plantago $(5,96 \%)$, Urticáceas $(5,43 \%)$ y Casuarina $(2,54 \%)$.

Por otra parte (fig. 4), se puede apreciar que, prácticamente durante todo el año, predomina el polen de los taxones leñosos sobre el de los herbáceos, excepto durante los meses de Agosto y Septiembre, en los que hay una ligera predominancia de éstos últimos, aunque hay que tener en cuenta que durante esta época las concentraciones polínicas son muy bajas. El predominio de los tax ones leñosos está ocasionado por especies arbóreas como olivo, Cupresáceas y Quercus que, por otra parte, son los taxones que mayor incidencia tienen en la atmósfera.

\section{DISCUSIÓN}

En general, puede decirse que el comportamiento del polen total ha sido similar 


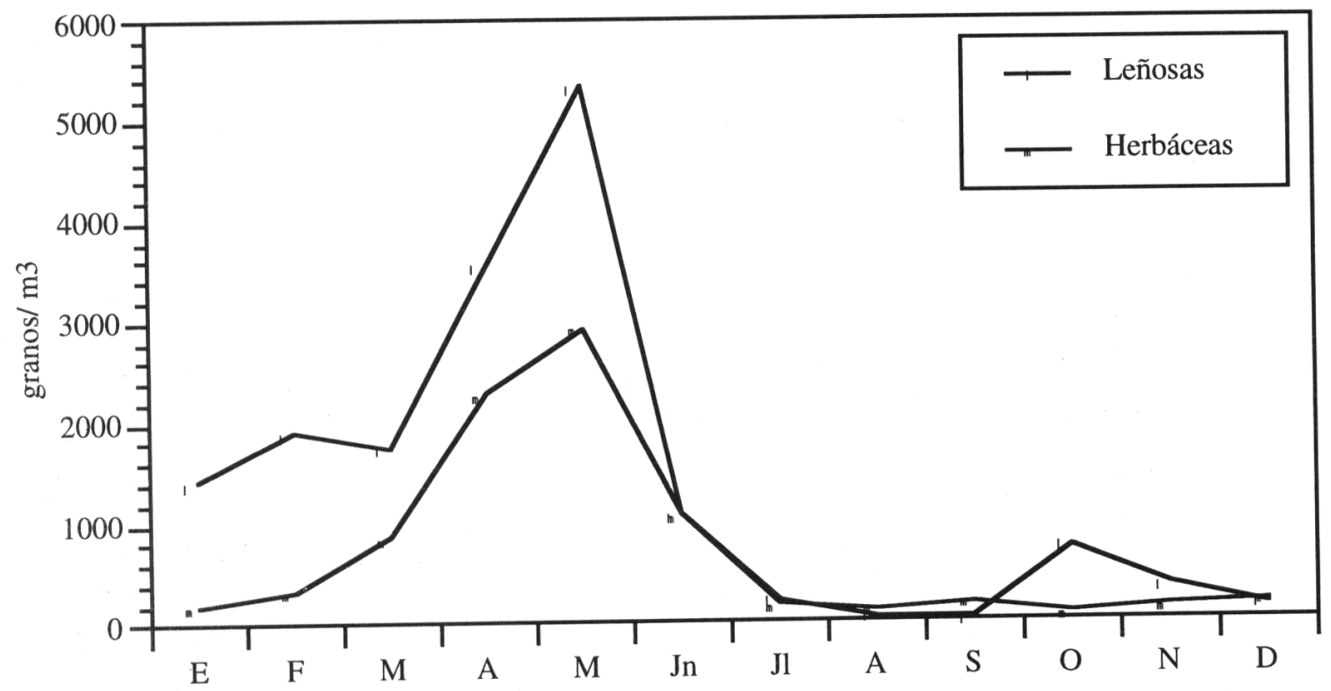

Figura 4. Distribución mensual de las concentraciones obtenidas por el polen de las especies leñosas frente al de herbáceas a lo largo de 1994. Total monthly values obtained by the pollen of plants classified into two groups: woody (trees and shrubs) and herbaceous (grasses and weeds) species.

al obtenido para los años 1992 y 1993 , (Cabezudo et al., 1994), tanto en lo referente a los valores totales anuales como al período en que se detectan las máximas concentraciones, si bien se aprecia que el primer pico del año queda ligeramente desplazado hacia el mes de Enero, debido a que las concentraciones máximas de Cupresáceas se obtuvieron durante la última semana de este mes, lo que hizo que el recuento total mensual fuera muy superior al obtenido durante los años anteriores (tabs. 1 y 4). Por el contrario, se observa que, durante los meses de verano, las concentraciones totales mensuales quedaron por debajo de los resultados obtenidos en los dos años anteriores, probablemente debido a las elevadas temperaturas que se alcanzaron a principios de Julio (fig. 3), lo que, unido a la escasez de precipitaciones, impidieron el normal desarrollo de muchas especies.

Durante el año 1994, se ha observado un importante incremento del polen de especies herbáceas como gramíneas, Plantago y
Urticáceas y también, aunque algo más ligero, de Quercus, Rumex y compuestas, al tiempo que descendieron los valores totales anuales de Cupresáceas, Platanus y Artemisia, mientras que el resto de las especies se mantuvieron en valores más o menos similares (tab. 2).

En cuanto al momento en que se producen las máximas concentraciones, se observa que, con respecto a los dos años anteriores, los días pico registrados para los diferentes taxones, se han obtenido, en general, más tempranamente (tab. 4), si bien este adelanto ha sido más acusado en Cupresáceas, Pináceas, Quercus y Casuarina; y más ligero, aunque apreciable, en Plantago, Chenopodiáceas-Amarantáceas y gramíneas, por citar sólo los taxones más relevantes. La causa de esta precocidad en la fenología de las distintas especies, probablemente habría que buscarla, al menos en las especies herbáceas, en las escasas precipitaciones registradas durante la época primaveral, ya que durante el período MarzoJunio apenas se registraron $72 \mathrm{~mm}$ de 
precipitación (fig. 2), valor muy inferior a la media de los últimos 40 años. En este sentido, el olivo ha resultado ser una de las especies más estables, ya que es el taxón cuyo día pico ha presentado menos oscilaciones en los últimos tres años, produciéndose siempre en la primera decena del mes de Mayo (entre el día 2 y el 9).

Por último, hay que señalar que, en general, los niveles alcanzados por los picos máximos semanales, se mantienen en valores semejantes a los de los años 1992 y 1993, si bien hay que destacar las mayores concentraciones obtenidas por el polen de Quercus y, aunque menos acusadas, de Plantago y de gramíneas. Sin embargo, no sucede lo mismo con los valores registrados en los días picos, sensiblemente más elevados en 1994 especialmente en el caso de Cupresáceas y olivo, pero también en el de Urticáceas, Plantago, gramíneas, Mirtáceas y Casuarina.

AGRADECIMIENTOS. Los autores desean agradecer a las Consejerías de Salud y Educación de la Junta de Andalucía la colaboración prestada a la Red Andaluza de Aerobiología (RAA), para el desarrollo de este trabajo.

\section{BIBLIOGRAFÍA}

CABEZUdO, B., TRIGO, M. M., RECIO, M. y TORO, F. J. -1994- Contenido polínico de la atmósfera de Málaga: años 1992 y 1993. Acta Bot. Malacitana, 19: 137-144.

DOMÍNGUEZ VILCHES, E., GALÁN SOLDEVILLA, C., VILLAMANDOS DE LA TORRE, F. \& INFANTE GARCÍAPANTALEÓN, F. -1991- Handling and evaluation of the data from the aerobiological sampling. Monografías REA/EAN 1: 1-18.
GARCÍA GONZÁLEZ, J. J. -1995- Calendario polínico de la ciudad de Málaga. Prevalencia de tests cutáneos. Universidad de Málaga. Colección Tesis Doctorales, microfichas $\mathrm{n}^{\circ} 131$.

S.E.A.I.C. y ABELLÓ, S.A. -1995-Alergológica. Factores epidemiológicos, clínicos y socioeconómicos de las enfermedades alérgicas en España. Industrias gráficas Nilo, Madrid.

Aceptado para su publicación en Mayo de 1995

Dirección de los autores. Departamento de Biología Vegetal. Facultad de Ciencias. Universidad de Málaga. Apdo. 59. 29080, Málaga. Fax. (95) 21319 44. e-mail: Botánica@ccuma.sci.uma.es 